\title{
Satisfacción laboral y cultura organizacional en docentes universitarios en una Facultad de Ciencias de la Salud
}

\author{
Job satisfaction and organizational culture in university teachers in a Faculty of Health Sciences
}

Satisfação no trabalho e cultura organizacional em professores universitários de uma Faculdade de Ciências da Saúde

\section{Angélica Díaz Tinoco}

adiazt@unac.edu.pe

https://orcid.org/0000-0001-9915-3418

Universidad Nacional del Callao, Lima-Perú

Olga Giovanna Valderrama Rios

ogvalderramar@unac.edu.pe

https://orcid.org/0000-0003-1906-7101

Universidad Nacional del Callao, Lima-Perú

\author{
Alicia Lourdes Merino Lozano \\ amerinope@gmail.com \\ https://orcid.org/0000-0003-4430-0104 \\ Universidad Nacional del Callao, Lima-Perú \\ Llerme Nuñez Zarazú \\ opebri@yahoo.com \\ https://orcid.org/0000-0002-8011-5072 \\ Universidad Nacional Santiago Antúnez de Mayolo, \\ Ancash-Perú
}

\section{Juana Matilde Cuba Sancho \\ jcubas@unmsm.edu.pe \\ https://orcid.org/0000-0002-9803-1571}

Universidad Nacional Mayor de San Marcos, Lima-Perú

Artículo recibido 27 de septiembre 2021, arbitrado y aceptado 29 de octubre 2021 y publicado 30 de diciembre 2021

\section{RESUMEN}

Identificar la relación de la cultura organizacional en la satisfacción laboral de los docentes de una universidad de la costa peruana. Investigación descriptiva, correlacional y transversal. La muestra fue de 48 docentes, obtenidos mediante muestreo aleatorio simple. Los instrumentos utilizados fueron dos; la escala de satisfacción laboral SLSPC y otro para cultura organizacional, ambos en escala de Likert. Para los análisis estadísticos se usó la correlación de Spearman. El $66.7 \%$ presentó una cultura organizacional neutra, el $20.8 \%$ fuerte y el $12.5 \%$ débil. El $79.2 \%$ indicaron tener una satisfacción laboral media, $14.6 \%$ alta y solo el $6.3 \%$ baja. La cultura organizacional se relacionó con la satisfacción laboral de los docentes $(\mathrm{r}=0,375, \mathrm{p}=0,009)$.

Palabras clave: Cultura Organizacional; Satisfacción laboral; Docentes; Universidad

\section{ABSTRACT}

Identify the relationship of the organizational culture in the job satisfaction of the teachers of a university on the Peruvian coast. Descriptive, correlational and cross-sectional research. The sample consisted of 48 teachers, obtained by simple random sampling. The instruments used were two; the SLSPC job satisfaction scale and another for organizational culture, both on a Likert scale. Spearman's correlation was used for statistical analyzes. $66.7 \%$ presented a neutral organizational culture, $20.8 \%$ strong and $12.5 \%$ weak. $79.2 \%$ indicated having a medium job satisfaction, $14.6 \%$ high and only $6.3 \%$ low. Organizational culture was related to teachers' job satisfaction $(r=0.375, p=0.009)$.

Key words: Organizational culture; Work satisfaction; Teachers; University

\section{RESUMO}

Identificar relação da cultura organizacional na satisfação profissional de professores de uma universidade do litoral peruano. Pesquisa descritiva, correlacional e transversal. A amostra foi constituída por 48 professores, obtida por amostragem aleatória simples. Os instrumentos usados foram dois; a escala de satisfação no trabalho SL-SPC e outra de cultura organizacional, ambas em escala Likert. A correlação de Spearman foi usada para análises estatísticas. 66,7\% apresentaram cultura organizacional neutra, $20,8 \%$ forte e $12,5 \%$ fraca. $79,2 \%$ indicaram ter uma média de satisfação no trabalho, $14,6 \%$ alta e apenas $6,3 \%$ baixa. A cultura organizacional esteve relacionada à satisfação profissional dos professores $(\mathrm{r}=0,375, \mathrm{p}=0,009)$.

Palavras-chave: Cultura organizacional; Satisfação no trabalho; professores; Universidade 


\section{INTRODUCCIÓN}

El estudio de la cultura organizacional es de gran valor fundamentalmente porque permite apreciar el trabajo a los docentes, generando un impacto en la motivación, satisfacción y producción del docente de la educación superior, por lo tanto, son las autoridades quienes deben realizar programas de desarrollo para que los docentes puedan potenciar sus competencias profesionales de ese modo puedan fortalecer sus valores y aumentando la retención de los buenos maestros. Una buena organización cuya base esté en valores y normas permite a cada uno de los miembros identificarse con la institución manteniendo conductas positivas e incrementando su productividad dando una buena imagen del lugar donde laboran, es decir, se logra un exitoso sistema de gestión.

La cultura organizacional a nivel académico implica la provisión de un servicio más que un producto. Los clientes de las organizaciones académicas se centran en los estudiantes donde es demostrado que la cultura organizacional tiene un impacto no solo en la investigación, sino incluso en la adopción de métodos de enseñanza innovadores.

Las universidades como centro principal y clave para la educación de los seres humanos, tienen una cultura especial como las demás organizaciones que puedan jugar un papel significativo en la formación de personas cualificadas, expertas y emprendedoras, por lo que requiere la existencia de una cultura organizacional adecuada en las Instituciones de Educación Superior. Estos centros preparan a los jóvenes para realizar cambios constantes en la sociedad y en el mundo, por lo que, la universidad tiene el enorme compromiso de responsabilidad cultural sobre su carga como institución cultural y creadora de cultura.
Pedraza, Obispo, Vásquez y Gómez (2015) definen la cultura organizacional se define como los supuestos básicos sobre el mundo y los valores que guían la vida en las organizaciones. Actualmente, las organizaciones afrontan diferentes fenómenos tal es el caso de la globalización la que tiene como consecuencia una alta complejidad del contexto global produciendo un impacto en los procesos sociales afectando el desarrollo de las personas en el ámbito laboral y por ende en la satisfacción laboral.

Las Instituciones de Educación Superior enfrentan grandes retos e induce grandes efectos sobre la capacidad para satisfacer con su función social lo que demanda garantizar atención especial a los diferentes procesos que desarrollan y a su gestión (Pedraza et al., 2015), lo cual demanda a la vez elevar la calidad de la planificación, la implementación y el control de la estrategia institucional mencionan Rodríguez, De León y Galarza (2015).

Desde el punto de vista de la Psicología (Pedraza et al., 2015) las declaraciones subjetivas de bienestar, en general, y de satisfacción laboral, en particular, han mostrado sus bondades como herramientas para el análisis del bienestar subjetivo de las personas (Nosnik, 2005). En este contexto se observó que los docentes de la Facultad de Ciencias de la Salud de la Universidad Nacional del Callao desde el punto de vista de la cultura organizacional, hay un desconocimiento de los principios y valores de la Universidad, se presentan inconformismos por ciertos manejos laborales, que se realizan en la organización en cuanto al desarrollo del talento humano o que puede generar dificultades en el cumplimiento de su misión y visión. 
La satisfacción laboral de los profesores universitarios está relacionada con una serie de variables de función compleja, como los caracteres demográficos, el trabajo en sí mismo, el salario, las responsabilidades laborales, la variedad de tareas, las oportunidades de ratificación y promoción, la relación con los compañeros de trabajo y otros. Los profesores pueden estar simultáneamente satisfechos con algunas facetas del trabajo e insatisfechos con otros.

Es importante conocer cómo perciben los docentes el desarrollo de la propia cultura organizacional necesaria para cumplir con la misión y visión institucional para así elevar el nivel de la calidad educativa propiciando un clima de trabajo confortable, por lo que es necesario un estudio de investigación que permita interpretarla desde el punto de vista de sus miembros, como un primer acercamiento para que la organización educativa reflexione sobre sus futuras decisiones, acertadas o no, tomando en consideración las razones que inducen a su personal para pensar de esa manera y tomar una postura frente a su ambiente laboral.

En este contexto, la presente investigación identificar la relación existente entre la relación entre la cultura organizacional y la satisfacción laboral de los docentes de una universidad pública de la costa peruana.

\section{MÉTODO}

El presente fue un estudio de enfoque cuantitativo, descriptivo, correlacional y transversal. La muestra fue de 48 profesores de la Facultad de Ciencias de la Salud de la Universidad Nacional del Callao en Perú de una población de 60, quienes aceptaron participar de manera voluntaria en el estudio. La técnica utilizada para la recolección de datos fue la encuesta, donde el instrumento para satisfacción laboral fue elaborado por Palma (2005), esta escala utiliza la técnica de Likert, consta de 27 ítems. Para la variable cultural organizacional, se usó el instrumento elaborado por Olmos (2006). Fueron modificadas y validadas mediante un juicio de expertos, así como la confiabilidad fue evaluada mediante la prueba de Alfa de Cronbach, en la que se obtuvo un valor de 0,80 , lo que indica que ambos instrumentos son fiables para la obtención de información y su alta consistencia interna. La modalidad de envío del cuestionario fue virtual relacionado al contexto de la pandemia en la que se ha vivenciando, y la recolección de datos fue durante el mes de setiembre del 2020. Se solicitaron los permisos a las autoridades de la Facultad y al comité de ética institucional.

Para el análisis y procesamiento de datos se utilizó el software estadístico SPSS 26.0 y se hizo uso de la estadística descriptiva e inferencial. Para determinar la asociación de las variables se tuvo en cuenta la correlación de Spearman. Asimismo, se respetó el derecho al anonimato y confidencialidad de los datos de los participantes.

\section{RESULTADOS}

La mayoría de los docentes $41.7 \%$ tuvo una edad comprendida entre 51 y 60 años, el $75.0 \%$ fueron de sexo femenino, el $77.1 \%$ fue casado, el $45.8 \%$ era docente a tiempo parcial y trabajaba 20 horas mientras que el $43.8 \%$ de docentes tenía 21 años y más de tiempo de servicios (Gráfico 1). 
Con respecto a la cultura organizacional de los docentes de la Universidad Nacional del Callao, el 67\% de los docentes evidenciaron una cultura organizacional neutra, el $21 \%$ tuvo una cultura organizacional fuerte, mientras que el $13 \%$ evidenciaron una cultura organizacional débil (Gráfico 2).
Delos 48 docentes encuestados, el79\% indicaron tener una satisfacción laboral media, seguido del $15 \%$ docentes que presentaron una satisfacción laboral alta y sólo el $6 \%$ docentes presentaron una satisfacción laboral baja (Gráfico 3).

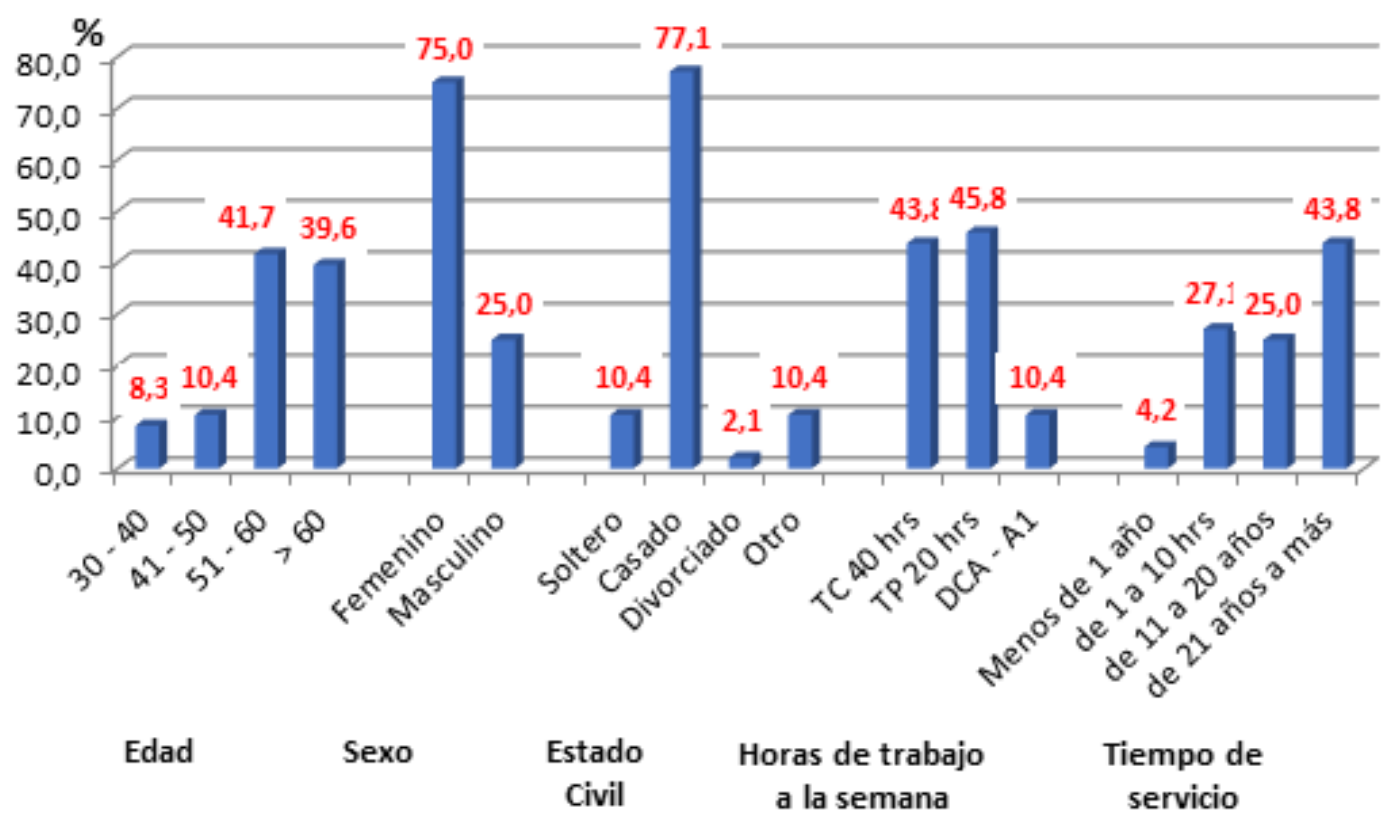

Gráfico 1. Características generales de los docentes de una universidad de la costa peruana.

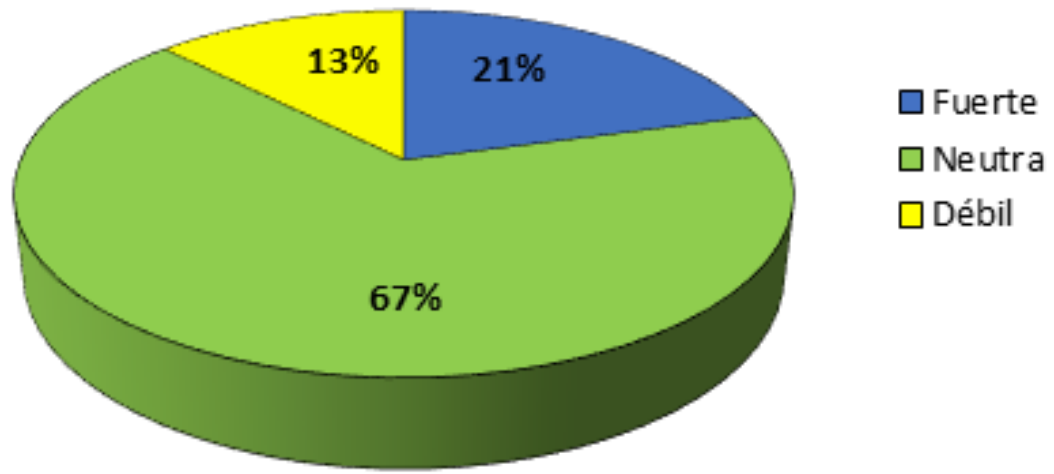

Gráfico 2. Cultura organizacional de los docentes de una universidad de la costa peruana. 


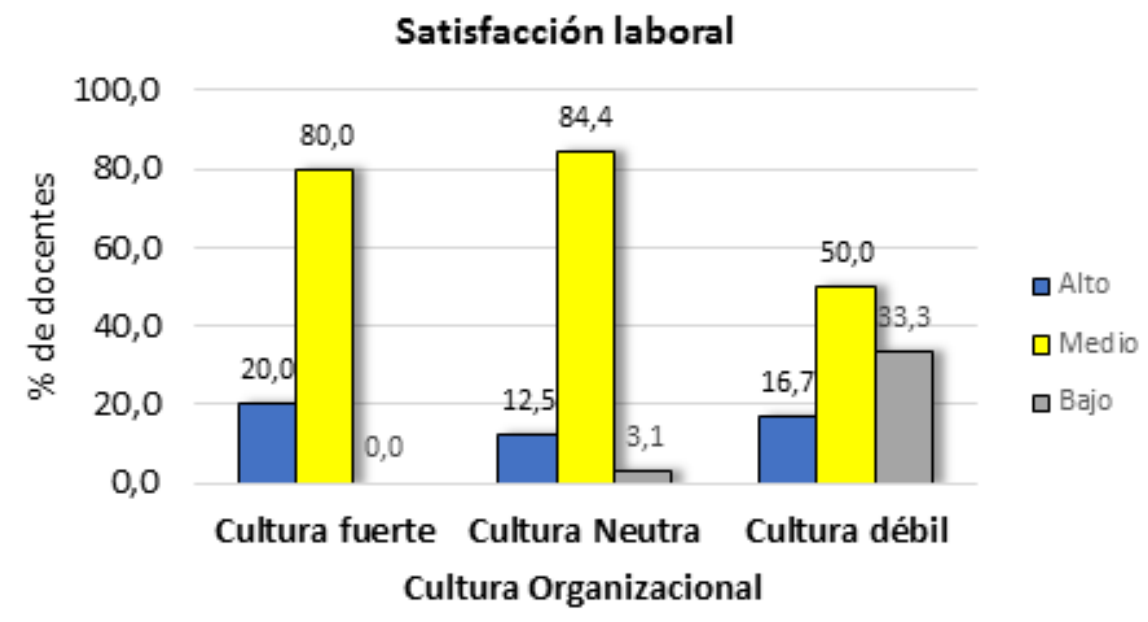

Gráfico 3. Porcentaje de docentes de una universidad de la costa peruana por satisfacción laboral, según cultura organizacional.

Se aprecia que del total de docentes que presentaron una cultura organizacional fuerte, el $80 \%$ presentó un nivel de satisfacción medio, el $20 \%$ presentó un nivel de satisfacción alto y ninguno presentó un nivel de satisfacción bajo. Del total de docentes que presentó una cultura organizacional neutra el $84.4 \%$ presentó un nivel de satisfacción laboral medio, el $12.5 \%$ presentó un nivel de satisfacción alto y solo el $3.1 \%$ presentó un nivel de satisfacción bajo. Del total de docentes que presentó una cultura organizacional débil, el 50.0\% presentó un nivel de satisfacción medio, el $33.3 \%$ presentó un nivel de satisfacción bajo y solo el 16.7\% presentó un nivel de satisfacción alto. Esto con un análisis correlacional estadístico, determinó que la cultura organizacional se relacionó con la satisfacción laboral de los docentes, obteniendo un valor de 0,375 para una p de 0,009 , ver Gráfico 4 .

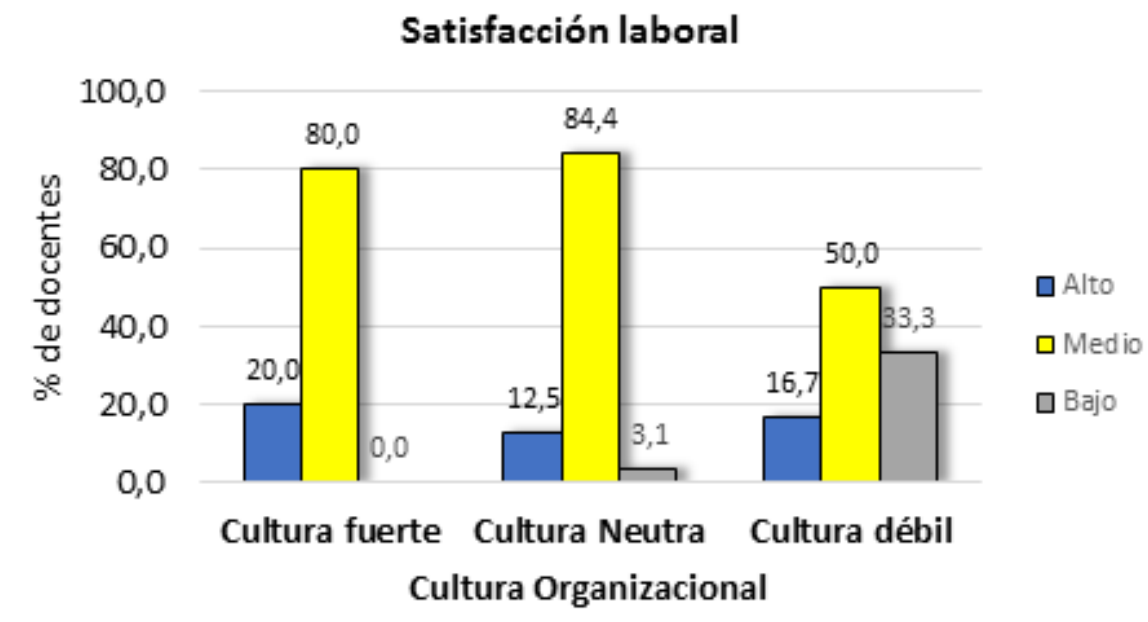

Gráfico 4. Porcentaje de docentes de una universidad de la costa peruana por satisfacción laboral, según cultura organizacional. 


\section{Discusión}

Los resultados demuestran que la cultura organizacional influye en la satisfacción laboral de los docentes de la Facultad de Ciencias de la Salud de la Universidad Nacional del Callao. Estos resultados concuerdan con Durand (2015) demuestra que existe una relación significativa entre cultura organizacional y satisfacción laboral, además refiere que existe una moderada relación positiva. Por otra parte, Ibidunni y Agboola (2014) refieren que existe una cultura "correcta" o "mejor" para todas las organizaciones. La cultura más apropiada para una organización es la que mejor le ayuda a hacer frente a las exigencias de su entorno empresarial. Asimismo, Yuang, Jia y Zhao (2016) encontraron que la identificación organizacional moderaba la asociación entre la cultura organizacional y la satisfacción laboral. Encontrando puntajes más altos en satisfacción laboral que aquellos con baja cultura organizacional. Con respecto a la cultura organizacional de los docentes, el $66.7 \%$ cuentan con una cultura organizacional neutra, el 20.8\% cuentan con una cultura organizacional fuerte $y$ el $12.5 \%$ cuentan con una cultura organizacional débil.

Con respecto a la variable satisfacción laboral, Alfaro, Leyton, Meza y Sáenz (2012) encontraron que los trabajadores tienen un nivel de satisfacción laboral medio; y que existen diferencias significativas por condición laboral respecto al nivel de satisfacción en sus diversos factores en cada una de ellas. Datos que concuerdan con el presente estudio donde se encontró que el 79.2\% indicaron tener una satisfacción laboral media, seguido del $14.6 \%$ de docentes que presentaron una satisfacción laboral alta y solo el 6.3\% de docentes presentaron una satisfacción laboral baja.
Por otra parte, Muñoz (2019) encontró una correlación significativa y positiva entre las dimensiones de la variable cultura organizacional y los factores de satisfacción laboral. Cotocel, Lovan, Gogănău, Nechita, Deca, Cotoi y otros (2019) en los resultados de su estudio demostraron que los docentes se encuentran satisfechos con la organización donde se respeta y reconoce los méritos profesionales, asimismo la satisfacción laboral ya que se respeta las oportunidades de desarrollo, Cheung y Lun (2015) examinaron la asociación entre la satisfacción laboral y cultura organizacional. La correlación bivariada mostró que tanto la actuación profunda como la expresión de emociones sentidas naturalmente estaban relacionadas positivamente con las dos dimensiones de satisfacción laboral.

$\mathrm{Al}$ respecto, en el presente estudio se encontró que los docentes que presentaron una cultura organizacional fuerte, el $80 \%$ presentó un nivel de satisfacción laboral medio, el 20\% presentó un nivel de satisfacción laboral alto y ninguno presentó un nivel de satisfacción laboral bajo, del total de docentes que presentó una cultura organizacional neutra el $84.4 \%$ presentó un nivel de satisfacción laboral medio, el $12.5 \%$ presentó un nivel de satisfacción laboral alto y solo el 3.1\% presentó un nivel de satisfacción laboral bajo, del total de docentes que presentó una cultura organizacional débil, el 50.0\% presentó un nivel de satisfacción laboral medio, el $33.3 \%$ presentó un nivel de satisfacción laboral . 
CONCLUSIONES

La relación entre la cultura organizacional y la satisfacción laboral de los docentes del Facultad de Ciencias de la Salud de la Universidad Nacional del Callao, evidencian que existe una regular fuerza de relación entre dichas variables y que si la cultura organizacional se mejorará así también lo hará la satisfacción laboral.

\section{REFERENCIAS}

Alfaro, R., Leyton S., Meza, A. y Sáenz, I. (2012). Satisfacción laboral y su relación con algunas variables ocupacionales en tres municipalidades. http://tesis.pucp.edu.pe/repositorio/bitstream/ handle/

Cheung, F. y Lun, V. (2015). Satisfacción laboral y cultura organizacional: una investigación en educadores de China. J Gen Psychol, 142 (4), 253-72. http://dx.doi:10.1080 / 00221309.2015.1091764

Cotocel, C., Iovan, R., Gogănău, A., Nechita, F., Deca, S., Cotoi, B. y otros. (2019). Cultura organizacional e integración personal en la educación médica: estudio de caso Universidad de Medicina y Farmacia de Craiova, Rumania. Rom J Morphol Embryol, 60 (3), 1053-1058.https://www.ncbi.nlm.nih.gov/ pubmed/31912123

Durand, L. (2015). Cultura organizacional y satisfacción laboral del personal administrativo del Hospital Regional Huacho. http://repositorio. ucv.edu.pe/bitstream/handle/UCV/6434/Lino_ DLM.pdf? sequence $=1$ \&isAllowed $=y$
Ibidunni, O. y Agboola, G. (2014). Prediciendo el desempeño a través de los elementos de la cultura organizacional de seis universidades de Nigeria. Archives of Business Research, 2(6). http://dx.doi:10.14738/abr.26.736

Muñoz, V. (2019). Cultura organizacional y satisfacción laboral en los colaboradores de una empresa de servicios de entretenimiento. http:// repositorio.urp.edu.pe/handle/URP/2316

Nosnik, A. (2005). Culturas organizacionales: origen, consolidación y desarrollo. México

Olmos, M. y Socha, K. (2006). Diseño y validación mdeidate jueces expertos del instruemtno para evaluar cultura organizacional. Universidad de la Sabana. https://core.ac.uk/download/ pdf/47066084.pdf

Palma, S. (2005) Escala de satisfacción laboral (SLSPC). Lima: Cartolan E.I.R.L

Pedraza, A., Obispo, S., Vásquez, G., y Gómez, G. (2015). Cultura organizacional desde la teoría de Edgar Schein: Estudio fenomenológico. Clío América, 9 (17),17-25

Rodríguez, D., De León, L. y Galarza, J. (2015). Los retos actuales de las instituciones de educación superior en el área de la gestión. Revista Universidad y Sociedad, 7(3), 52-55. http://rus. ucf.edu.cu/

Yuan, G., Jia, L. y Zhao, J. (2016). Organizational Identification Moderates the Impact of Organizational Justice on Job Satisfaction.0 (0), 189-195. http://dx.doi: 10.3233/WOR-162271 